\title{
Unsupervised Change Detection in SAR images using Gaussian Mixture Models
}

\author{
E. Kiana ${ }^{\text {a }}$, S. Homayouni ${ }^{\text {b }}$ M.A Sharifi ${ }^{\text {a }}$ M. Farid-Rohanic \\ ${ }^{a}$ School of Surveying and Geospatial Engineering, Dept. of Remote Sensing, College of Engineering, U. of Tehran, Iran, \\ $\{$ kiana, sharifi\}@ut.ac.ir \\ ${ }^{\mathrm{b}}$ Dept. of Geography, Environmental Studies and Geomatics, U. of Ottawa, Ottawa, Canada, saeid.homayouni@uOttawa.ca \\ ${ }^{c}$ Faculty of mathematical Sciences, Dept. of Statistics, University of Shahid Beheshti, Iran, M_faridrohani@ sbu.ac.ir
}

KEY WORDS: Gaussian Mixture model, Change Detection, SAR images, Difference image, Multi-temporal Images

\begin{abstract}
:
In this paper, we propose a method for unsupervised change detection in Remote Sensing Synthetic Aperture Radar (SAR) images. This method is based on the mixture modelling of the histogram of difference image. In this process, the difference image is classified into three classes; negative change class, positive change class and no change class. However the SAR images suffer from speckle noise, the proposed method is able to map the changes without speckle filtering. To evaluate the performance of this method, two dates of SAR data acquired by Uninhabited Aerial Vehicle Synthetic from an agriculture area are used. Change detection results show better efficiency when compared to the state-of-the-art methods.
\end{abstract}

\section{INTRODUCTION}

Land changes, caused by climate change our human activity, can be mapped automatically using multi-temporal satellite images (Celik, 2010). Change detection in remote sensing, is a process that analyses images of the same geographical area at different times in order to identify any changes which have taken place between the two acquisition dates (Celik, 2009). Being insensitive to atmospheric and illumination conditions, the Synthetic Aperture Radar (SAR) system has the advantages of being an all-day, all-weather device, as well as offering wide area coverage (Curlander and McDonough, 1991). As result, SAR images have more potential for detecting changes that are happened during the time. Change detection procedures may be classified in two groups, pixel and object based approaches. Object based approaches is the best tools for high resolution optical images. SAR images usually, have low spatial resolution. As result, pixel based approaches are recommended for these active earth observation data. Several change detection pixel based methods have been proposed, e.g. Image differencing, Image rationing, Regression analysis, Vegetation index differencing, Change vector analysis (CVA), Principal component analysis (PCA), Tasselled cap transformation (TK), Texture analysis based, post classification comparison, Multidate direct comparison, Artificial Neural Network, Support Vector Machine, Decision Tree, GIS integration, Spectral mixture analysis, Fuzzy change detection, Multi-sensor data fusion for change detection (Hussain et al., 2013) . In this paper, image differencing is used due its simplicity and easy to interpret results.

There are two categories of change detection methods in the remote sensing literature: supervised and unsupervised approaches. In supervised classification methods, a priori information is used as training data for the learning process of the classifiers (Bruzzone and Prieto, 2000). On the other hand, unsupervised classification methods work without training data through direct comparison of images (Bruzzone and Prieto, 2000). Supervised approaches have advantages of recognition of different land cover transitions and robustness to different conditions. But the appropriate training data generation for most of the applications, is usually an expensive and difficult task (Bruzzone and Prieto, 2000). As a result, in this paper the unsupervised strategy is used for change detection.
Automatic process doesn't exist for separate between change and no change pixels in the difference image (Bruzzone and Prieto, 2000). Such discrimination is usually performed by using empirical strategies or manual trial-and-error procedures, which affect both the accuracy and the validation of the changedetection process (Bruzzone and Prieto, 2000). In this paper we proposed a simple method for estimating a threshold that discriminates between changed and unchanged pixels in the difference image.

This paper is structured as follows. Section 2 introduces the unsupervised change detection problem and describes Gaussian mixture model of difference image and describes the proposed unsupervised change detection algorithm. Section 3 provides some testable results of the proposed method and compares with the state-of-the-art method presented in finally, the Section 4 conclude the paper.

\section{METHODOLOGY}

\subsection{Problem definition}

The proposed method contains mainly two steps:

1) Calculating the difference image from two precisely coregistered multi temporal images, and then image histogram is calculated.

Let's consider two satellite images, $\mathrm{X}_{1}=\{\mathrm{x} 1(\mathrm{r}, \mathrm{c}) \mid 1<\mathrm{r}<\mathrm{H}, 1<\mathrm{c}<\mathrm{w}\}$ and $\mathrm{X}_{2}=\left\{\mathrm{x}_{2}(\mathrm{r}, \mathrm{c}) \mid 1<\mathrm{r}<\mathrm{H}, 1<\mathrm{c}<\mathrm{W}\right\}$, of size $\mathrm{H}^{*} \mathrm{~W}$ pixels acquired from the same geographical area but at two different time instances, namely $t_{1}$ and $t_{2}$, respectively. Difference image $\left(x_{d}\right)$ can be calculated by the following equation:

$$
X_{d}(\mathrm{r}, \mathrm{c})=x_{2}(\mathrm{r}, \mathrm{c})-x_{1}(\mathrm{r}, \mathrm{c})
$$

2) Classification of difference image into three classes (i.e. negative change class, positive change class that named change class and no change class), using Gaussian Mixture Model (GMM). The unsupervised change detection procedure is based on mixture model. In statistics, a mixture model is a probabilistic model for representing the presence of subpopulations in an overall population, without needing that an observed data set should identify the sub-population to which an individual observation belongs. Mixture models are used to make statistical inferences about the properties of the sub-populations given only observations on the main population, without sub-population 
identity information. For change detection problem the parameters of each Gaussian distribution should be estimated.

\subsection{Gaussian mixture model of difference image}

$\mathrm{K}$ distributions with density functions $\mathrm{f}_{1}(\mathrm{x}), \ldots, \mathrm{f}_{\mathrm{K}}(\mathrm{x})$ can be mixed in proportions $\pi 1, \ldots, \pi_{\mathrm{k}}$ to give mixture distribution with density function:

$$
f(x)=\sum_{k=1}^{K} \pi_{k} f_{k}(x)
$$

When data items are real vectors, it may be reasonable to model the data as a mixture of Gaussian distributions, using the data to estimate both the mixing proportion and the mean vector and covariance matrix of each component distribution. This model, with K components, can be written as:

$$
f(x)=\sum_{k=1}^{K} \pi_{k} \varphi\left(\frac{x-\mu_{k}}{\sigma_{k}}\right)
$$

where $\mu_{k}$ and $\sigma_{k}$ 's are the mean and standard deviation of Gaussian distributions, respectively. By using the data, the estimate of the mixing proportions and the mean and variance of each Gaussian components can be obtained. A natural idea of estimation of $\pi_{k}, \mu_{k}$ and $\sigma_{k}$ for $\mathrm{k}=1, \ldots, \mathrm{K}$ is maximizing the likelihood function. Assuming that data items are independent, the log likelihood is

$$
\sum_{i=1}^{N} \log f\left(x_{i}\right)
$$

Where $x_{i}$ is the data vector for item $i$, and $f(x)$ is as defined in equation (3). One can use different general purpose optimization methods in order to find the parameters of a mixture model that maximize the likelihood. However, the EM algorithm is commonly used, thanks to its simplicity of implementation and its stability.

If one knows which mixture component each data item came from, the estimating mixing proportions and the parameters of each component distribution are straightforward. However, in general, the user does not have this knowledge. By giving an initial guess of the parameters, one can probabilistically assign a component to each data item, and then get a better estimate of the parameters based on these devolution. This idea can be similar to C-means algorithm, but in a probabilistic setting, with a proof that the algorithm will attain a (local) maximum of the likelihood.

There are two general approaches for change detection from mixture distributions, that known as threshold-based and rule- based method. When mean and variance of each component are estimated, the thresholds can be calculated as the injection points of distributions. One of the rule-based method is the Bayes' rule that assign pixel $j$ in class $i$ if and only if

$$
\pi_{i} \varphi\left(\frac{x_{j}-\mu_{i}}{\sigma_{i}}\right)=\max _{k}\left\{\pi_{k} \varphi\left(\frac{x-\mu_{k}}{\sigma_{k}}\right)\right\}
$$

When mean and variance of each component are estimated, the thresholds can be calculated as the injection points of distributions. In the histogram of difference image, pixels before first threshold are negative changes (change pixels), pixels between two thresholds are no changes and pixels after second threshold are positive change pixels (change pixels). Figure 1 demonstrates an overview of proposed method.

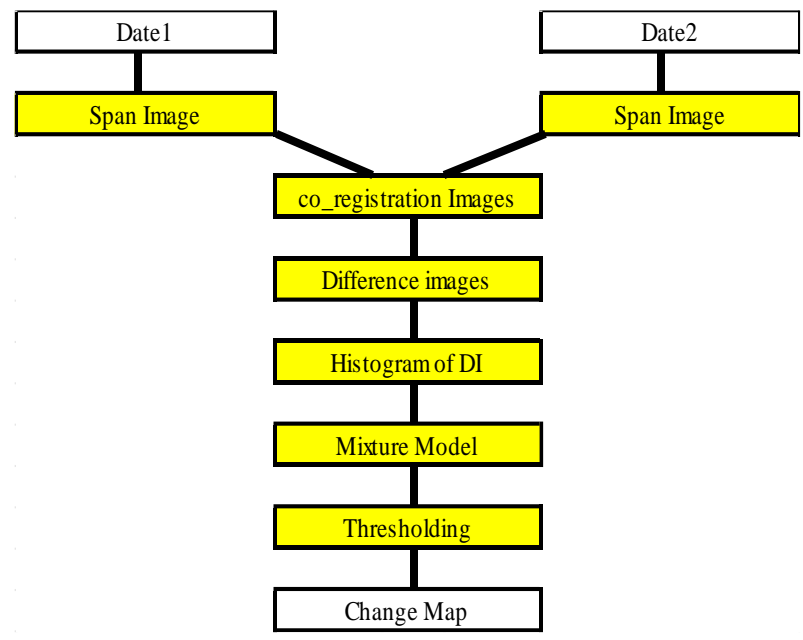

Figure 1. An overview of the proposed method

In Figure 4 the difference image as shown in Fig. 3(c) is used to demonstrate estimating the difference image data distribution using Equation (4).

In preprocessing step, the span image (i.e. the total power of multi-polarization intensities) of each date was calculated from covariance matrix. Two images are, then, co-registered with high accuracy. Histogram of difference image has nearly Gaussian distribution as shown in Figure 2. In this histogram, the no change pixels are close to the mean value and the changed pixels fall in two end of distribution(Jensen and Lulla, 1987). The proposed method, estimates the threshold in histogram of difference image. 


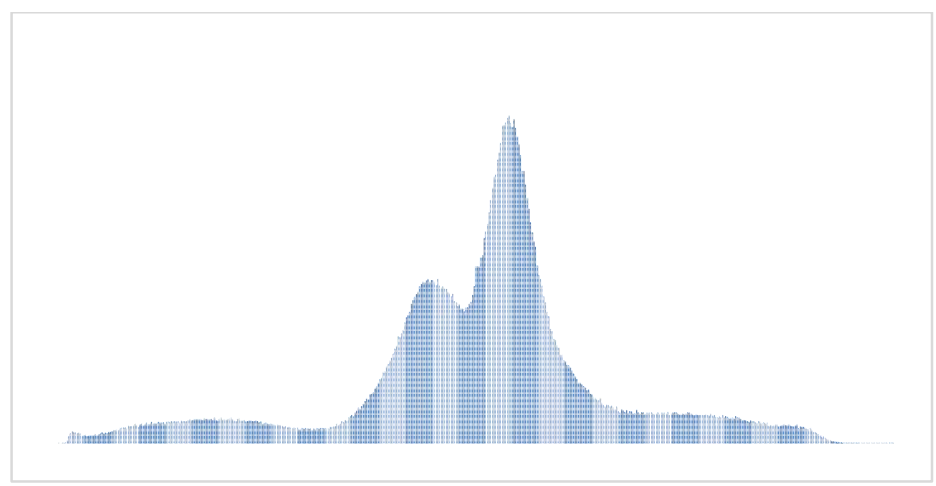

Figure 2. Histogram of differencing image

a)

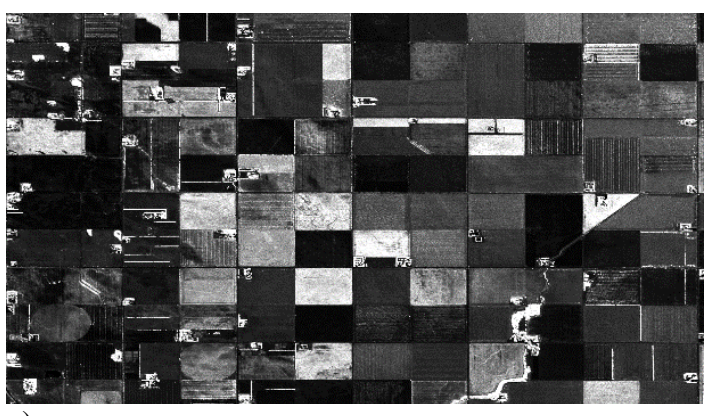

c)

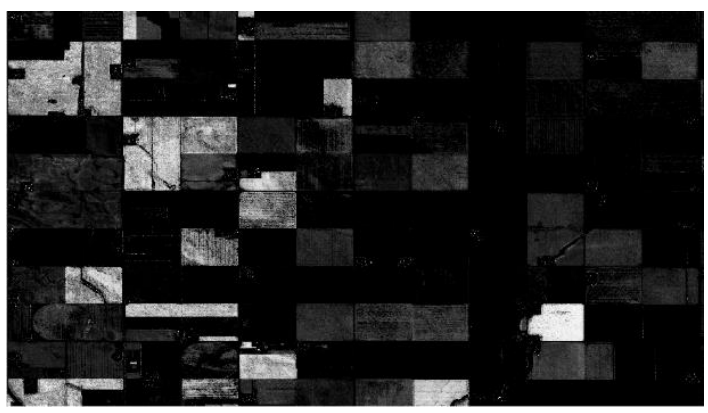

b)

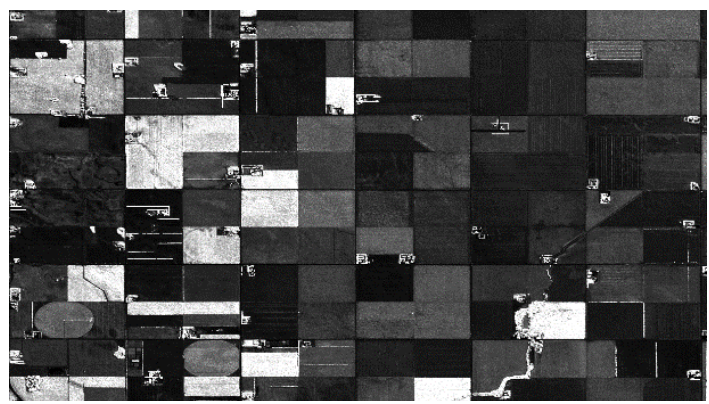

d)

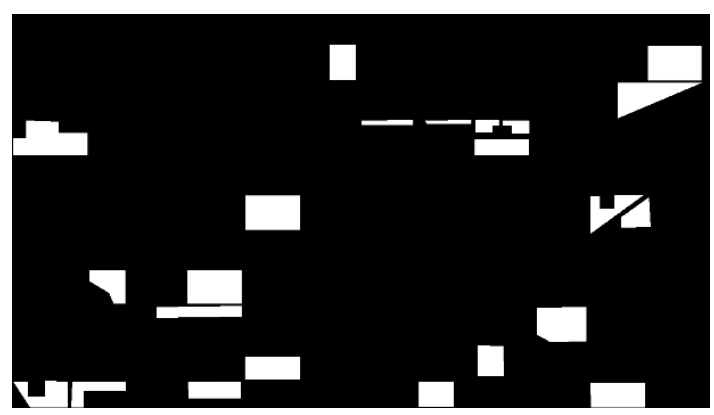

Figure 3.First Span Image (a), Second Span Image (b), Difference Image (c) Ground Truth (d).

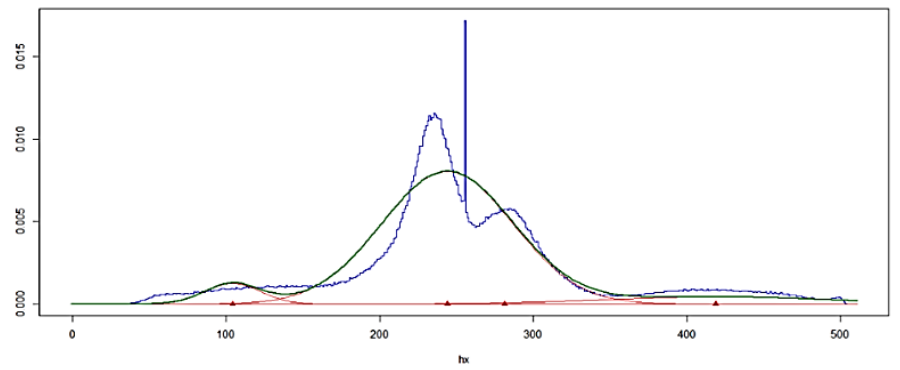

Figure 4.Difference image data modelling and estimating data distributions of "changed" and "unchanged" pixels of the difference image as shown Figure 3(c)

\section{SAR DATA PROCESSING}

\subsection{Description of dataset}

The proposed method was applied to two dates of polarimetric SAR data acquired by Uninhabited Aerial Vehicle Synthetic Aperture Radar (UAVSAR) L-band system. The UAVSAR radar has a range bandwidth of $80 \mathrm{MHz}$ which gives it a $1.8 \mathrm{~m}$ range resolution(Rosen et al., 2006).

The dataset contains 699 by 1237 pixels selected from agricultural area near Winnipeg, in Manitoba, Canada, during Jane and July in 2012 (See Figure 3.a and 3.b). The difference image of two dates is calculated by equation (1) and is presented in Figure 3.c. The ground truth of the change detection mask was created by a visual analysis of the input images (See Figure 3.d). 


\subsection{Primary Results}

In this paper comparisons between the proposed method and Otsu's methods occurred by the ground truth of the change detection mask. As showed in Figure 5, there are pixels that detected as changed pixels (white and gray color) in Gaussian mixture model but in Otsu model they are detected as no changed pixels. Changed pixels in Gaussian mixture model are classify in two clusters. First cluster contains the pixels with high brightness in first image and low brightness in the second image. This may explain that high brightness is surface scattering and low brightness is volume scattering agricultural field. Second cluster is those pixels that have low brightness in first image and high in the second one. As shown in Table1, the overall accuracy of proposed method is $82 \%$, Kmeans model is $58 \%$ and Otsu method is $38 \%$ these method compare with the ground truth of the change detection mask as shown in figure 3(d). Since Otsu's method calculates all the components, it needs more time to detect the change map.

\begin{tabular}{|l|c|c|c|}
\hline & Proposed Model & Kmeans Model & Otsu Model \\
\hline Overall accuracy & $\mathbf{8 2 \%}$ & $\mathbf{5 8 \%}$ & $\mathbf{3 8 \%}$ \\
\hline
\end{tabular}

Table1 demonstrate Overall accuracy of Proposed model, Kmeans model and Otsu model

a)

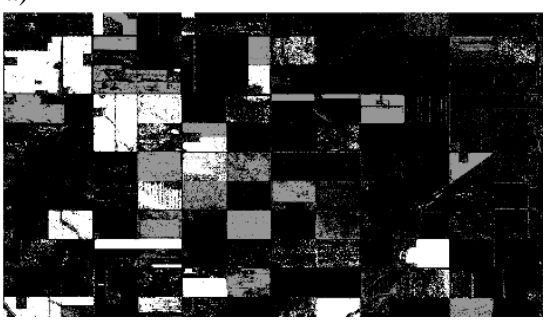

b)

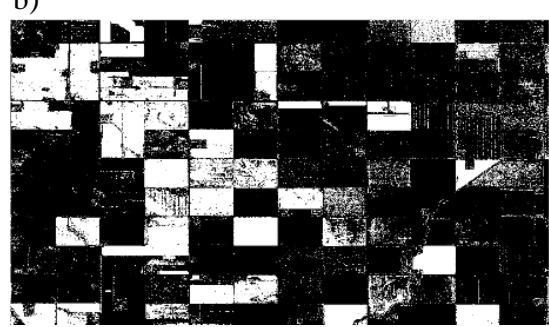

c)

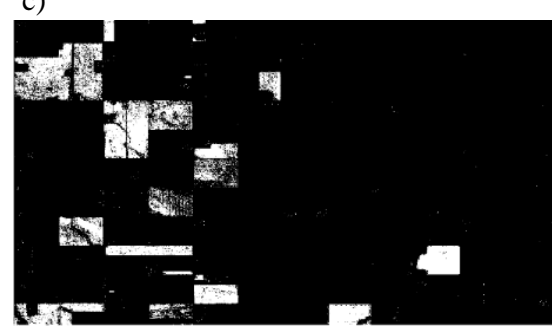

Figure 5. Change map resulted by proposed Mixture model (a) change map obtained by Kmeans model (b) change map obtained by Otsu model (c).

\section{CONCLUSIONS}

In this paper, we proposed a novel method for unsupervised change detection in multi-temporal SAR images of the same scene using Gaussian Mixture model (GMM). The Gaussian mixture model is adopted to sort the difference image. In this process, the difference image is classified into three classes; negative change class, positive change class and no change class. The proposed method have high accuracy in front of Otsu method

\section{REFERENCES}

Bruzzone, L., Prieto, D.F., 2000. Automatic analysis of the difference image for unsupervised change detection. Geoscience and Remote Sensing, IEEE Transactions on 38, 1171-1182.

Celik, T., 2009. Unsupervised change detection in satellite images using principal component analysis and-means clustering. Geoscience and Remote Sensing Letters, IEEE 6, 772776 .

Celik, T., 2010. Image change detection using Gaussian mixture model and genetic algorithm. Journal of visual communication and image representation 21, 965-974.

Curlander, J.C., McDonough, R.N., 1991. Synthetic aperture radar. John Wiley \& Sons. and Kmeans method proposed method do not spend more time for detect change map. However the SAR images suffer from speckle noise, the proposed method is able to map the changes without speckle filtering. The proposed method is comfortable for agricultural field with a lot change in their crops.

As we know, the difference image histogram of optic images follow the Gaussian distribution so we can implement proposed method into optic images too.

Hussain, M., Chen, D., Cheng, A., Wei, H., Stanley, D., 2013. Change detection from remotely sensed images: From pixelbased to object-based approaches. ISPRS Journal of Photogrammetry and Remote Sensing 80, 91-106.

Jensen, J.R., Lulla, K., 1987. Introductory digital image processing: a remote sensing perspective.

Rosen, P., Hensley, S., Wheeler, K., Sadowy, G., Miller, T., Shaffer, S., Muellerschoen, R., Jones, C., Zebker, H., Madsen, S., 2006. UAVSAR: A new NASA airborne SAR system for science and technology research, Radar, 2006 IEEE Conference on. IEEE, p. 8 pp. 